\title{
Pulmonary hydatid embolization Report on 2 operated cases and review of published reports
}

\author{
Abraham Palant, Victor Deutsch, Yehezkiel Kishon, Yair Lieberman, Joseph H. Yahini, \\ and Henry N. Neufeld \\ From the Heart Institute, Department of Diagnostic Radiology and Thoracic Surgery, Chaim Sheba Medical \\ Center, Tel Hashomer, and the Sackler School of Medicine, Ramat-Aviv, Israel
}

Two patients with pulmonary hydatid embolization are described and commented upon in the light of 43 similar published cases. The diagnosis was strongly suspected from the medical history and the chest $\mathrm{x}$-ray films and supported by angiocardiography. The angiocardiographic features of this condition have not been described previously in detail. They include amputation and filling defects of pulmonary artery branches, which are typically located proximal to a rounded tumour-like opacity seen on the plain x-ray film. Both patients underwent successful embolectomy.

Pulmonary hydatid embolization is a rare complication of cardiac or hepatic echinococcosis. A review of the world medical reports reveals only 43 cases of this condition, most of them diagnosed at necropsy (Purriel et al., 1970). Only two were studied by angiocardiography and in none was a successful pulmonary embolectomy performed.

This report describes two patients with pulmonary hydatid embolization. Both underwent angiographic studies and were relieved by pulmonary embolectomy.

\section{Case 1}

\section{Case reports}

This 42-year-old Arab woman, born in Israel, had undergone laparotomy for removal of an hepatic hydatid cyst at the age of 22 . In June 1971, she was admitted to another hospital with the chief complaints of pain in the right upper quadrant and right lower chest, cough, and shortness of breath.

On physical examination she was very breathless and there was mild cyanosis. Blood pressure was $120 / 80 \mathrm{mmHg}$. The pulse rate was $80 / \mathrm{min}$, regular. On auscultation only a faint apical systolic murmur was heard. The right lower lung was poorly ventilated. The liver was palpated 2 to $3 \mathrm{~cm}$ below the right costal margin and was not tender. There was

Received 16 March 1976. no pitting oedema of the extremities. The chest $x$-ray film showed a right-sided pleural effusion. Routine laboratory findings were normal except for the differential blood count which showed 10 per cent eosinophils. A diagnosis of pulmonary embolism was made and anticoagulant therapy instituted. Her condition improved rapidly and she was discharged in satisfactory general state. However, she was readmitted three months later with an identical picture, suggestive of recurrent pulmonary embolization. Progressive and refractory heart failure soon developed. On the eleventh day in hospital, her condition deteriorated further with increasing dyspnoea, right-sided chest pain, and a critical fall in blood pressure. The possibility of a massive pulmonary embolization was then considered and the patient transferred to the Chaim Sheba Medical Center for further evaluation and possible operation.

On admission her general condition was poor. She was in severe respiratory distress with moderate cyanosis. The heart rate was $100 / \mathrm{min}$, and the blood pressure $100 / 70 \mathrm{mmHg}$. The neck veins were much engorged. A right ventricular heave was palpated. A grade $2 / 6$ systolic ejection murmur was heard along the left sternal border. The second sound in the pulmonary area was widely split with a loud pulmonary component. Expiratory râles and a pleural friction rub were heard over 


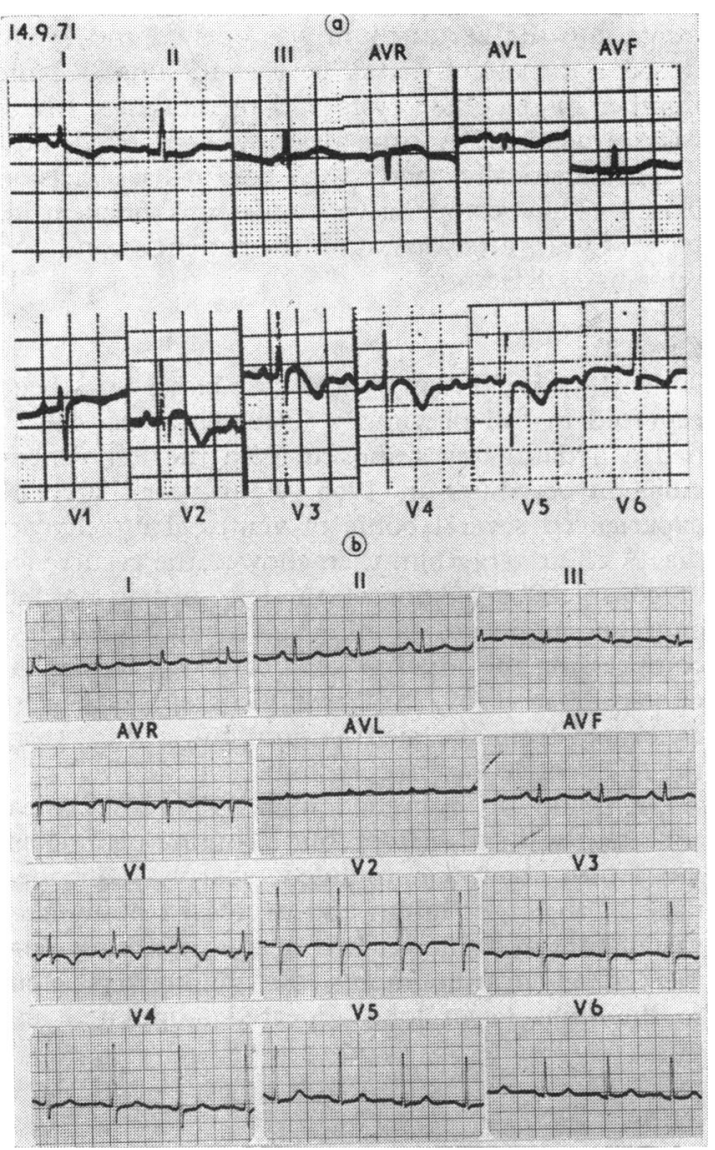

FIG. 1 Case 1. Preoperative electrocardiogram (a) showing right ventricular overload (inverted $T$ in V1-V6) with postoperative regression (b).

the right lung field. The liver was felt 4 fingerbreadths beneath the right costal margin and was tender. The spleen was not palpable. There was no ascites or pitting oedema of the lower extremities.

The electrocardiogram showed sinus rhythm, right axis deviation, and incomplete right bundlebranch block, with symmetrically inverted $\mathrm{T}$ waves in the right chest leads and clockwise rotation (Fig. 1a). The chest $x$-ray film showed moderate cardiomegaly and a rounded sharply demarcated mass at the right hilum (Fig. 2). The pulmonary vasculature of the right lower and middle lobes was decreased and a ring of calcification was seen within the liver.

These findings suggested massive pulmonary embolization. Furthermore, in view of the past history of hepatic echinococcosis and the presence of a calcified annular shadow in the liver region,

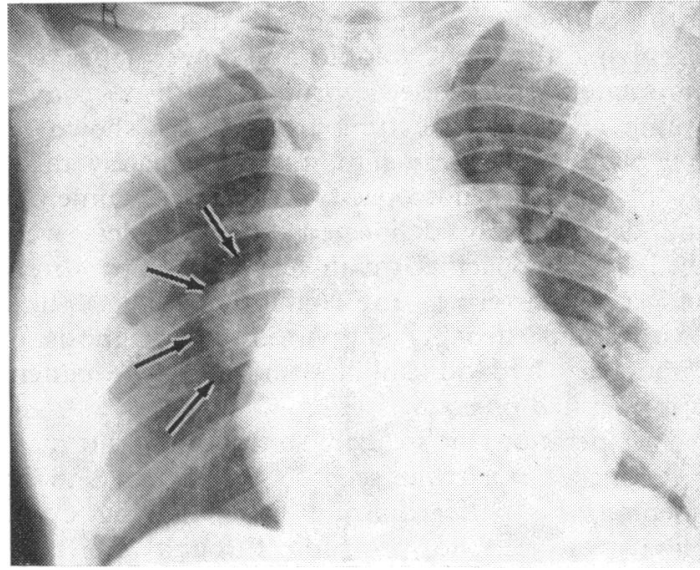

FIG. 2 Case 1. Chest X-ray film, showing a round, sharply demarcated mass at the right hilum (arrows) with decreased vascularity of the right base.

the possibility of pulmonary hydatid embolization was strongly considered. An emergency right heart catheterization was then performed which disclosed a pulmonary arterial pressure of $58 / 30$ (mean 24) $\mathrm{mmHg}$, right ventricular pressure of $60 / 5 \mathrm{mmHg}$, and right atrial pressure of $8 \mathrm{mmHg}$ (mean).

Selective injection of contrast material into the

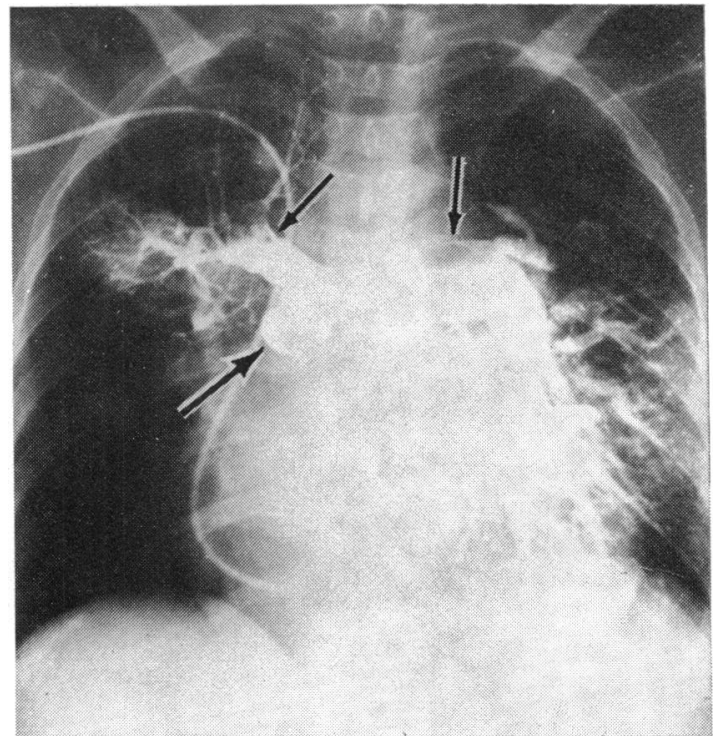

FIG. 3 Case 1. Pulmonary angiogram, showing amputation of the intermediate branch of the right pulmonary artery (arrow kead), multiple rounded filling defects in the left pulmonary artery (arrows), and amputation of the apico-posterior branches of both right and left upper lobe arterics. 
main pulmonary trunk showed that the branch supplying the right middle and lower lobes was amputated and ended abruptly with a convex stump (Fig. 3). The tumour-like mass shown on the plain chest $x$-ray film was immediately distal to this, and did not opacify. Moreover, numerous rounded, sharply delineated filling defects were seen in the opacified main left pulmonary artery and in the artery to the right upper lobe. These observations strongly supported the diagnosis of pulmonary hydatid embolization, and the patient was operated on soon after.

At operation by median sternotomy, the main pulmonary artery was seen to be dilated, and on opening the pericardium a large bulging cystic mass was seen to protrude through the right diaphragm into the pericardial space. The pericardium and the myocardium were intact with no signs of cyst rupture. Cardiopulmonary bypass was instituted and a longitudinal incision made in the main pulmonary trunk up to its bifurcation. A large number of hydatid cysts and membranes were aspirated from the left and right pulmonary arteries as far distally as possible, by suction and irrigation with saline. At the end of this procedure backflow of blood was obtained from both pulmonary arteries. The main pulmonary trunk was closed by a running suture and extracorporeal circulation stopped. The cyst which was seen to bulge into the pericardium was opened and found to be a hepatic hydatid cyst containing a large number of daughter cysts and membranes, which were evacuated. A tube drain was inserted subdiaphragmatically and the pericardial incision closed. The postoperative course was uneventful. Now, 4 years after operation the patient's condition remains satisfactory.

\section{Case 2}

This 31-year-old man, whose case history has been reported in full previously (Deutsch et al., 1969), had a hydatid cyst removed from the left ventricular myocardium in 1965. Four years later he experienced several bouts of ventricular tachycardia. A chest $x$-ray film then showed the recurrence of conspicuous bulging of the left cardiac border, and on angiocardiography a mediastinal mass compressing the superior vena cava was shown. At operation in 1969, two echinococcal cysts were evacuated from the mediastinum and a third from the left ventricular myocardium.

On follow-up examination in 1974, his clinical condition was satisfactory but a routine plain chest $x$-ray film showed a rounded, sharply delineated mass at the right hilum and decreased pulmonary vasculature of the lower half of the right lung (Fig. 4). On angiocardiography, the pulmonary artery to the right lower lobe appeared amputated and

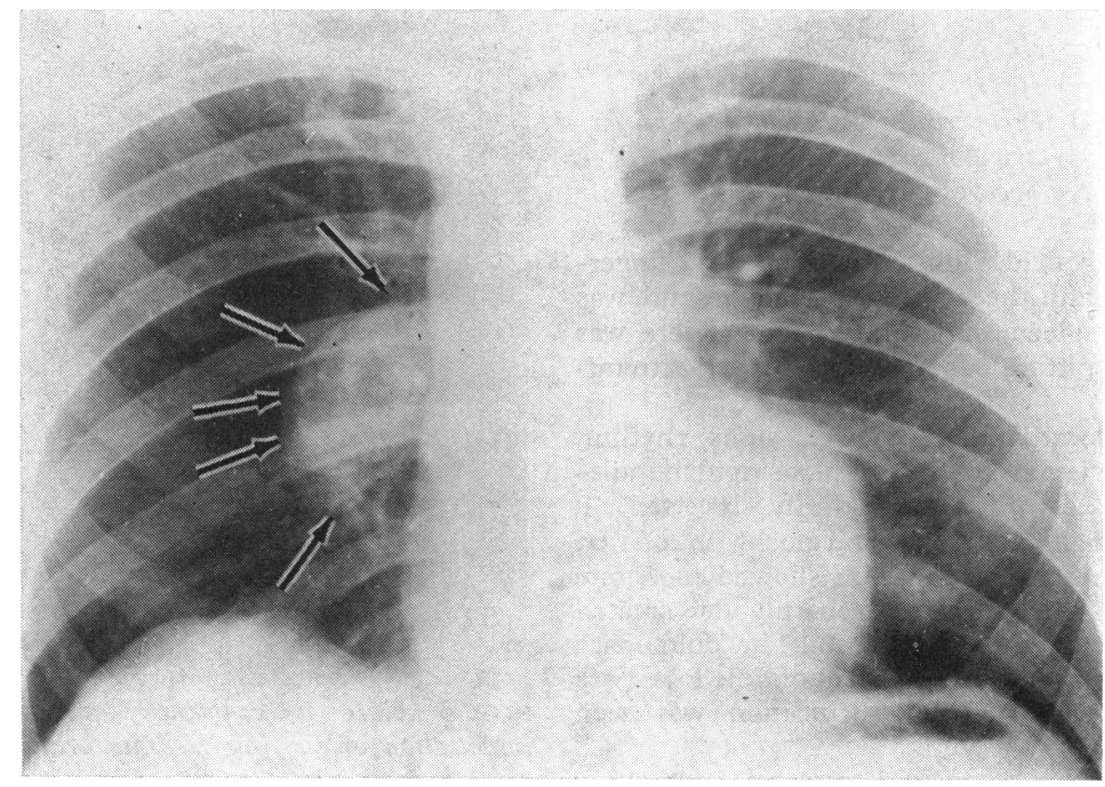

FIG. 4 Case 2. Chest x-ray film, showing a large round mass at the right hilum (arrows) with decreased vascularity of the right lung. The bulging left heart border is due to a myocardial scar after cardiotomy for cardiac echinococcosis. 


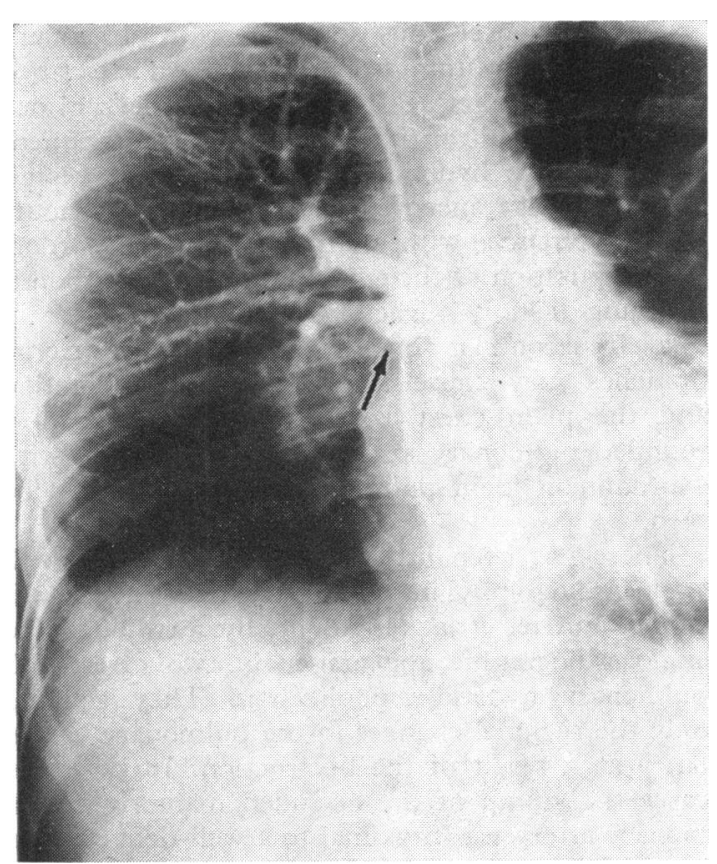

F I G. 5 Case 2. Pulmonary angiogram. Amputation of the intermediate branch of the right pulmonary artery proximal to the hilar mass (arrow).

ended abruptly with a convex contour. The pulmonary artery branches to the right upper and middle lobes and the left lung appeared to be normal (Fig. 5). The tumour-like mass on the plain chest $x$-ray film was seen to be distal to the occluded pulmonary artery stump and did not opacify.

From the patient's past medical history and our experience with the first case pulmonary hydatid embolization was diagnosed. At operation by right lateral thoracotomy, with division of multiple adhesions following previous operations, a large hydatid cyst, measuring $5 \mathrm{~cm}$ in diameter, was found at the right hilum. After the host membrane had been opened, the cyst was emptied with a trocar, and numerous daughter cysts and membranes were evacuated. Another cyst was found emerging from the intermediate part of the right pulmonary artery causing complete obstruction. On evacuation of the cyst, bleeding from the proximal part of the artery occurred and was controlled by suturing the arterial wall which was very thin and fragile. A huge hydatid cyst of the liver was then evacuated transdiaphragmatically and a drainage tube introduced below the diaphragm which was sutured.

The postoperative course was uneventful and five months later the patient remains in an excellent condition.

\section{Discussion}

A primary hepatic or cardiac hydatid cyst is always unilocular and contains only liquid and microscopical elements, namely the brood capsules, scolices, and the products of their disintegration (Deve, 1928). When such a cyst ruptures, it rarely gives rise to mechanical complications, but subsequent growth of the disseminated fragments of the germinal layer of the hydatid cyst may result in visceral echinococcosis. Once ruptured, the cyst may degenerate, die, or give rise to daughter cysts. Pulmonary hydatid echinococcosis occurs when a cyst containing daughter cysts and membranes ruptures and empties its contents into the lesser circulation.

Intracardiac rupture occurred in 104 of the 269 cardiac cysts reviewed by di Bello and Menendez (1963). Only 27 of these presented post-mortem evidence of pulmonary hydatid echinococcosis, in 24 as a result of intracavitary rupture of a rightsided cardiac hydatid cyst and in 3 from direct rupture into the pulmonary artery. Intracavitary rupture occurred in 35 of 94 cysts located in the left ventricle $(37 \%)$ compared with 30 of 34 located in the right ventricle $(80 \%)$. Thus, right ventricular cysts ruptured into the ventricular cavity in a much higher percentage than those located in the left ventricle, presumably because of the much thinner ventricular wall of the former and the higher pressure within the latter.

Purriel et al. (1970) recently reviewed 43 reported cases of pulmonary hydatid embolization, with clinical documentation in 33, including 4 of his own. In 29, the source of hydatid embolization was a ruptured cardiac cyst and in 14, a ruptured hepatic cyst into the inferior vena cava or hepatic vein. Purriel et al. (1970) also showed that pulmonary hydatid embolization may occur in distal branches of the pulmonary artery. This may result in an aneurysmal dilatation of the vessel, with thinning out of its wall; eventually the cyst may break through the vessel wall and invade the bronchi or lung tissue.

Another mechanism of pulmonary hydatid embolization is erosion of a mediastinal cyst with direct penetration into one of the pulmonary arterial branches causing embolization and obstruction of the latter. This form of pulmonary hydatid embolization has been documented previously in one patient from this hospital (Kreisler et al., 1965). This was a 21-year-old woman born in Iraq, who was admitted with clinical signs of severe 
pulmonary hypertension. A week after angiocardiography she suddenly collapsed and died in shock, death appearing to result from massive pulmonary hydatid embolization. At necropsy, the main pulmonary artery was surrounded with echinococcal cysts which compressed the artery and penetrated its wall. One of the cysts protruded into the arterial lumen. Another cyst was ruptured resulting in embolization and occlusion of the pulmonary arterial branches.

Both our cases had signs of hepatic echinococcosis. The first had no evidence of right heart echinococcosis, either at angiocardiography or at surgery. We assume, therefore, that pulmonary hydatid embolization was the result of a hepatic cyst rupturing into the hepatic veins or directly into the inferior vena cava. In the second case, the origin of the hilar cyst and of the pulmonary artery obstruction was difficult to trace at operation. A hepatic origin could not be excluded.

The clinical picture of pulmonary hydatid embolization is similar to one of ordinary pulmonary thromboembolism. In acute massive macroembolization by large echinococcal cysts and macroscopical hydatid elements, occlusion of the main pulmonary arteries may lead to acute cor pulmonale or sudden death. This occurred in 17 of the 33 cases with clinical documentation collected by Purriel et al. (1970). In less severe forms there may be a clinical picture of subacute cor pulmonale with raised venous pressure and decreased cardiac output. The latter was present in 3 of 33 cases mentioned above; all died within 3 to 10 months. In 13 cases, repeated rupture of a hydatid cyst resulted in recurrent pulmonary hydatid embolization. In the latter, chronic cor pulmonale developed in the form of progressive pulmonary hypertension and congestive heart failure of prolonged duration ( 2 to 10 years). In our first case the initial episode of hydatid embolization presumably caused only moderate obstruction of the pulmonary vasculature with a clinical picture of subacute cor pulmonale which subsided gradually. Subsequent embolizations, however, were life-threatening and necessitated prompt embolectomy. In our second case, pulmonary vascular obstruction by hydatid cysts occurred without apparent clinical manifestations.

The electrocardiogram in pulmonary hydatid embolization shows the same changes as in ordinary pulmonary thromboembolisms. Commonly there are symmetrically inverted $T$ waves of right ventricular systolic overload in right praecordial leads (Purriel et al., 1970). This was seen in our first case in whom the right ventricular strain pattern regressed after operation (Fig. 1b).
The plain chest $x$-ray appearances in pulmonary hydatid embolization which have been described recently (Purriel et al., 1970) were also seen in our cases. As a result of the impaction and subsequent growth of the hydatid cysts, the affected vessels tend to display aneurysmal dilatation and tumourlike appearance, with sharply delineated borders and amputation of their branches; the corresponding lung field is oligaemic. Trapping of hydatid cysts in second or third order pulmonary arterial branches may cause local aneurysmal dilatation and the plain chest $x$-ray film may then show rounded pulmonary shadows. Cardiomegaly, with a prominent main pulmonary trunk, may also be seen.

There has been little previous experience with angiocardiography in pulmonary hydatid embolization. Purriel et al. (1970) briefly mentioned the angiocardiographic appearance in two cases with pulmonary hydatid embolization. They stressed only the regional changes in the pulmonary vascularity as a result of the obstruction. In both our cases the stump of the occluded main right pulmonary artery was proximal to a well-defined hilar mass. Furthermore, in our first case, multiple, rounded, polypoid filling defects were seen in the main left pulmonary artery and the branches to the apicoposterior portion of the left upper lobe. In addition, the artery to the posterior segment of the upper lobe was also occluded and ended abruptly with a concave demarcation line.

However, these angiographic features are not pathognomonic for pulmonary hydatid embolization as ordinary pulmonary thromboembolism may cause similar findings. Furthermore, hilar bronchial carcinoma or mediastinal malignant disease may also present as a hilar mass distal to an occluded pulmonary artery. Also intracavitary right ventricular tumours may propagate into the pulmonary artery and cause similar polypoid filling defects. However, in patients with a history of hydatid involvement in other organs or from endemic areas of hydatid disease, angiography can be of great diagnostic value. In these circumstances, the first clue to the diagnosis of pulmonary hydatid embolization may be seen on the plain chest $x$-ray film, as happened in both our cases. Early diagnosis, particularly in the massive form of pulmonary hydatid embolization, is of major importance because surgical intervention could be life saving.

\section{References}

Deutsch, V., Kreisler, B., Padeh, B., and Pausner, Y. M. (1969). Echinococcosis of the heart diagnosed by cardioangiography. British fournal of Radiology, 42, 540. 
Dévé, F. (1928). -Les kystes hydatiques du coeur et leurs complications. Algerie Médicale, 32, 179.

Di Bello, R., and Menendez, H. (1963). Intracardiac rupture of hydatid cysts of the heart. A study based on three personal observations and 101 cases in the world literature. Circulation, 27, 366.

Kreisler, B., Padeh, B., Pausner, J., Deutsch, V., Bubis, H. J., and Kariv, Y. (1965). Echinococcus of the heart. Proceedings of the Tel-Hashomer Hospital, 4, 66.
Purriel, P., Tomalino, D., Muras, O., and Acosta Ferreira, W. (1970). Embolismo pulmonar hidatico. Estudio sobre 4 observaciones personales y revision de la literatura. Torax, 19, 164.

Requests for reprints to Professor Henry N. Neufeld, Chaim Sheba Medical Center, Tel Hashomer and Sackler School of Medicine, Tel Hashomer, Israel. 